\title{
Identificação de Geometrias Implícitas na Arquitetura a Partir de Realidade Aumentada
}

\author{
Alignment Identification of Implicit Geometries in Architecture from Augmented Reality
}

\author{
Tássia Borges de Vasconselos \\ Universidade Federal de Pelotas, Brasil \\ tassiav.arq@gmail.com \\ Adriane Borda Almeida da Silva \\ GEGRADI/DAURB/FAURB/UFPel, Brasil \\ adribord@hotmail.com
}

\begin{abstract}
For the initial stages of architectural training one of the strategies traditionally used is to study design cases. This exercise can help in the comprehension of implicit geometries and design processes. The use of the golden ratio, for example, is recurrent in the history of architecture. However, adequate instruments are necessary for this kind of statement. This paper demonstrates the use of Augmented Reality with this goal: virtual elements are viewed overlaying the architecture or its representation, configuring a "digital proportion measurer". The comprehension of known architectural practices assists in structuring methods and constructing repertoires for architectural design.
\end{abstract}

Keywords: Architecture analysis; Geometry; Augmented reality; Digital proportion measurer.

\section{Introdução}

A prática projetual na arquitetura, principalmente a partir de interesses didáticos, é estudada sob diferentes abordagens. Algumas delas buscam compreender como se dá esse processo, destacando para a necessidade de explicitá-lo, tal como a dada por Silva (1984). Este autor considera que devem ser incorporados instrumentos lógicos que deem apoio às decisões de projeto, para que o processo projetual deixe de ser comparado a uma "caixa preta".

Considera-se que desvendar processos compositivos geométricos adotados em obras arquitetônicas reconhecidas é uma prática didática apropriada. Além de explicitar estratégias lógicas e auxiliar na estruturação de métodos projetivos colabora na construção de repertórios para o projeto arquitetônico (Borda, Pires e Vaconselos, 2012)

Para a prática de arquitetura e, como consequência, para o processo de aprendizagem desta prática, a atividade de análise de casos de projeto se constitui como uma ação fundamental para aquisição de um repertório de estratégias para a configuração espacial. Desta maneira o projetista constrói sua própria memória, de acordo com a teoria da memória dinâmica (Schank, 1982, in: Heylighen,2000).

O estabelecimento de relações determinadas, de proporções, entre as partes de uma obra é um processo compositivo recorrente ao longo da história da arquitetura. Em muitos casos estas relações permitem explicar a configuração da forma de obras que se estabeleceram como referências de arquitetura Entretanto, a identificação do emprego destas relações não é algo evidente, como pode ser o emprego de simetrias por reflexão. Fazse necessário o uso de ferramentas adequadas para este tipo de análise.

Apoiando-se em métodos tradicionais de traçado, diversos autores (Doczi, 1990, Ching, 1998, Clarck \& Pause, 1987, Elam, 2001) demonstram a construção de conhecimento arquitetônico através da análise do emprego do conceito de proporção, sobre inúmeras obras de arquitetura.

Nesta direção e partindo-se de um interesse didático, considera-se a importância da inserção de práticas de análise formal em estágios iniciais de formação em arquitetura. Estas práticas permitem envolver conhecimentos prévios de geometria resgatados do ensino médio e que podem ser facilmente ampliados. Desta maneira, desde um primeiro momento de formação pode-se promover a compreensão dos estudantes sobre o que pode vir a ser um método projetual de arquitetura, pelo menos em termos formais. (Pires et al, 2011).

Entretanto, no contexto de formação em arquitetura que se insere este trabalho, tradicionalmente e de maneira objetiva, o uso de técnicas de análise formal utilizando-se do conceito de proporção era tratado em estágios avançados de teoria e projeto. Realizavase a construção de proporcionômentros em uma disciplina de Estética situada no 7ํㅗ semestre de formação. Eram distribuídos 
fachadas de edifícios históricos da cidade de Pelotas, e sobre elas, utilizando-se de tal instrumento, os estudantes analisavam as relações estabelecidas entre os elementos destas representações.

A construção destes proporcionômetros tradicionais em contexto educativo foi identificada também junto a uma disciplina optativa, situada no 5 o semestre de formação, da Faculdade de Arquitetura e Urbanismo da UFRGS (http://estratgen.blogspot.com.br/2009/04/aula-02-sintaxe-daforma.html).

A partir de 2011, fruto de uma reestruturação curricular, foi possível no contexto da FAURB/UFPel, trazer o mesmo tipo de exercício para o primeiro semestre de formação, na disciplina de GGD I, como exemplificado pela figura 1.

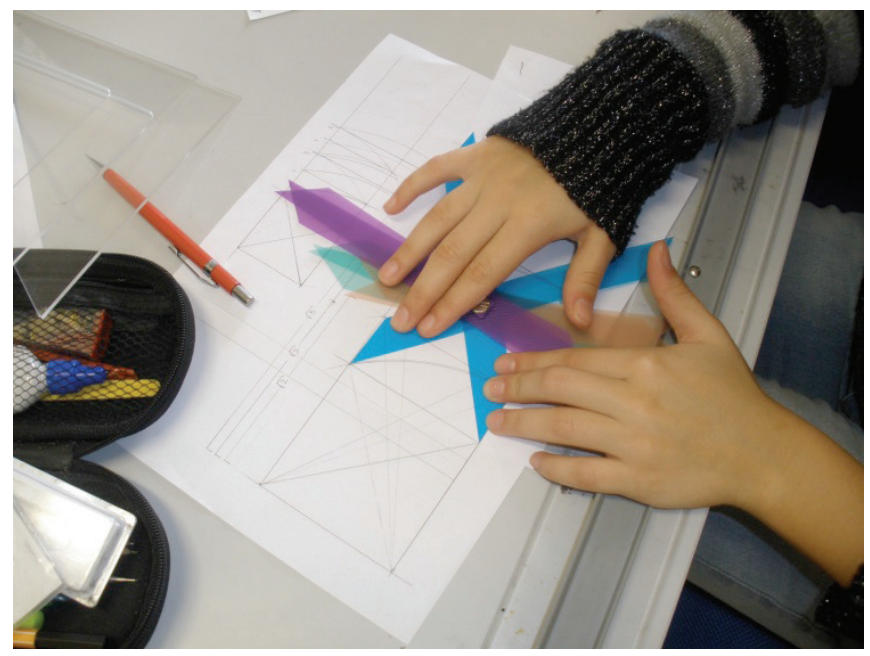

Figura 1: ilustração do uso do proporcionômetro junto à disciplina de GGDI/DAURB/FAURB/UFPel/2ㅇsem./2011. Fonte: autoras.

Atualmente as tecnologias digitais disponibilizam técnicas que permitem a configuração do espaço digital para facilitar este tipo de análise de maneira precisa e ágil. Recursos de parametrização permitem, por exemplo, sobrepor figuras proporcionais para serem comparadas com determinadas configurações formais.

Alguns softwares de aplicação já disponibilizam como primitivas de programa o retângulo áureo. O SketchUp, por exemplo, quando se está criando um retângulo, indica quando se obtém tal proporção, da mesma maneira como indica determinadas posições relativas entre o elemento que se está criando com os eixos $x, y$ e $z$ (destacando com uma cor determinada). Encontrase também o retângulo áureo como primitiva em programas de desenho paramétrico, como é o caso do plugin Grasshoper disponível para Rhinoceros.

Preocupando-se com os aspectos didáticos, e observando-se a evolução das tecnologias digitais de visualização, associou-se a lógica deste tipo de análise geométrica, sob o conceito de proporção, com a lógica de funcionamento do que se estabeleceu como Realidade Aumentada (RA).
De acordo com Kirner e Kirner (2008) as técnicas de RA permitem sobrepor elementos virtuais à realidade. Permitem, sobretudo, interagir em tempo real com estes elementos para que se adaptem à forma e à escala dos objetos do mundo real.

Esta interação é possível de ser realizada manualmente por interfaces táteis. Com este discurso, a RA parece responder às necessidades de uma atividade de análise, sob o conceito de proporção, tal qual se estabeleceu no âmbito da arquitetura. Porém, prometendo adicionar inúmeras vantagens. A partir destas considerações, este trabalho descreve a configuração de um dispositivo digital, apoiado nas tecnologias de RA, o qual se denominou no âmbito deste estudo como "proporcionômetro digital".

\section{Metodologia}

O estudo foi estabelecido através das seguintes etapas:

\section{Etapa de revisão}

Nesta etapa, além do reconhecimento da importância do conceito de proporção para apoiar processos de análise de arquitetura, buscou-se particularizar o estudo quanto aos materiais e métodos empregados para este tipo de análise. Os principais trabalhos utilizados durante esta etapa foram: Sanz e Moratalla (1998), especialmente por trazer uma abordagem didática sistematizada sobre o tema; Doczi (1990), Ching (1998), Clarck \& Pause (1987) Elam (2001), cujo conjunto de análises apresentadas por eles constituiu uma demonstração do uso do método gráfico de sobreposição. Todos demonstram a sobreposição de polígonos envolventes (retângulos áureos, retângulos de raiz e quadrados) sobre projeções ortogonais de obras de arquitetura; Rocha (2011), juntamente com os demais autores citados, permite o reconhecimento do uso do conceito de proporção ao longo da história da arquitetura.

\section{Etapa de identificação de instrumentos de análise sob o conceito de proporção:}

Levin (1978) demonstra o uso do instrumento chamado "Golden proportion calipers" (Compasso de proporção áurea) para analisar elementos da natureza (figura 2, à esquerda). $\mathrm{E}$, de acordo com este autor, Joseph, 1954, já havia descrito tal instrumento, denominando "Golden section compasses".

Este tipo de compasso, de proporção áurea, possui em suas extremidades movimentos invariáveis de expansão e diminuição. Assim, enquanto o lado maior afere uma dimensão equivalente à "a" o lado menor afere uma medida equivalente à "b", Sendo que "b" será igual à medida de "a", multiplicada pela constante de valor 0,618. (Carrilho, 2007) Assim fica estabelecida uma relação proporcional fixa, ilustrada pelas imagens à direita da Figura 2. 

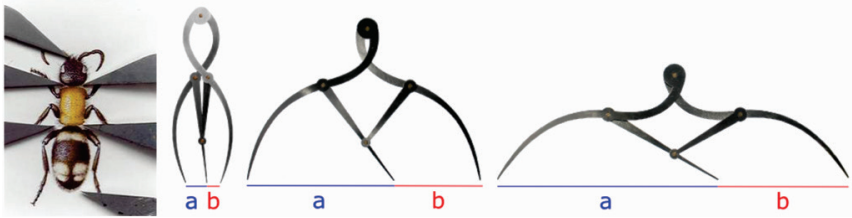

Figura 2: à esquerda: exemplo de medição com o compasso. Fonte: http://www.goldenmeangauge.co.uk/; à direita exemplo de um tipo de compasso, Fonte: http://www.goldenmeancalipers.com/.

De acordo com Krier (1993) esses compassos foram inventados pelos romanos. Mas sua patente, registrada em nome de Jack Nestor, data de 1988. O uso deste instrumento é bastante frequente na área de odontologia, especificamente na de ortodontia e dentística. Pode-se considerar tal compasso como uma versão industrial daquele utilizado em contextos de arquitetura. Entretanto, haveria a necessidade de ajustar as posições das conexões dos elementos retos ao longo das partes curvas. Com isto, o compasso não estaria restrito à medição da proporção áurea, incluindo qualquer outra prédeterminada, tal como o proporcionômetro tradicional.

Nesta etapa buscou-se, especialmente, identificar ferramentas e/ou dispositivos gráficos digitais que transpusessem a lógica do proporcionômetro tradicional ou do "compasso de proporção áurea".

Levin (2011) refere-se ao uso do software Phimatrix (http://www.phimatrix.com/). Esta ferramenta permite aplicar os conceitos de proporção aos estudos de estética dental destacado pelo autor, e também em outras áreas. Através dos meios digitais, facilita a sobreposição de traçados proporcionais sobre fotografias digitais dos sorrisos dos pacientes. Substituindo o método antigamente utilizado pelo autor que se utilizava da sobreposição de laminas transparentes com varias modulações.

No contexto trabalhado, no âmbito da disciplina de GGD I/DAURB/FAURB/UFPel, mencionada anteriormente, realizam-se os traçados no espaço virtual. Parte-se da digitalização das vistas e plantas baixas, utilizando-se de recursos de organização do espaço e de parametrização disponibilizados pelas ferramentas digitais de representação. Inicialmente, através da sobreposição de linhas que conectam ou tangenciam as formas representadas, identificam-se possíveis traçados reguladores. Os retângulos decorrentes destes traçados são analisados frente à correspondência com proporções determinadas.

Por outro lado, não foram encontradas referências ao uso de RA para o estabelecimento deste tipo de análise, mesmo na área de dentística.

\section{Etapa de desenvolvimento do proporcionômetro digital:}

O esquema da figura 3, explica a lógica de funcionamento do dispositivo de RA configurado para então permitir as atividades de análise. Retângulos virtuais, com proporções prédeterminadas, são sobrepostos à documentação de arquitetura ou mesmo diretamente sobre a visualização de edificações. Deve-se observar a necessidade de que a face do dispositivo móvel esteja paralela à superfície que se quer analisar. Para acionar a visualização em RA, uma câmera associada a um software processa a imagem obtida e identifica o símbolo, conhecido como QRcode, Quick Response code (código de resposta rápida), e disponibiliza o objeto virtual com base neste posicionamento.

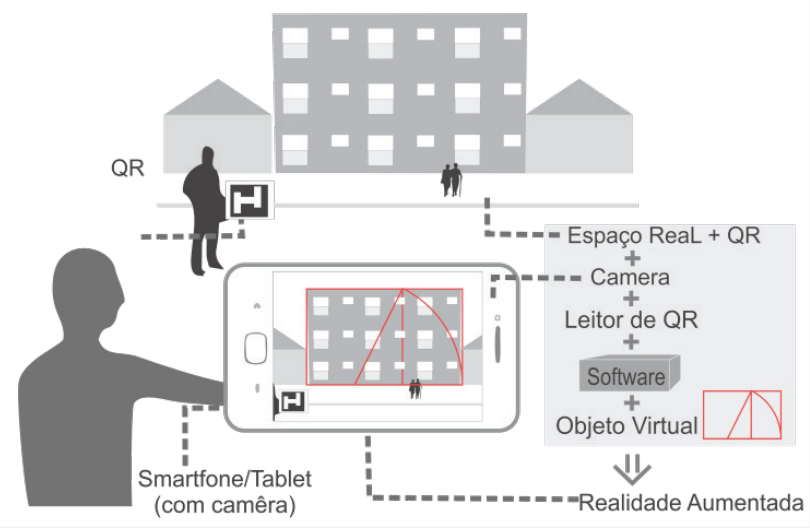

Figura 3: Esquema do funcionamento do dispositivo em RA. Fonte: autora.

O dispositivo foi estruturado a partir do aplicativo Augmented, o qual permite a visualização em RA e disponibiliza um repositório aberto e gratuito. Modelos de retângulo áureo, de raiz e quadrado foram disponibilizados neste repositório e podem ser utilizados através de smartphones ou tablets. A figura 4 ilustra, à esquerda, a interface do repositório e à direita o uso do proporcionômetro digital.

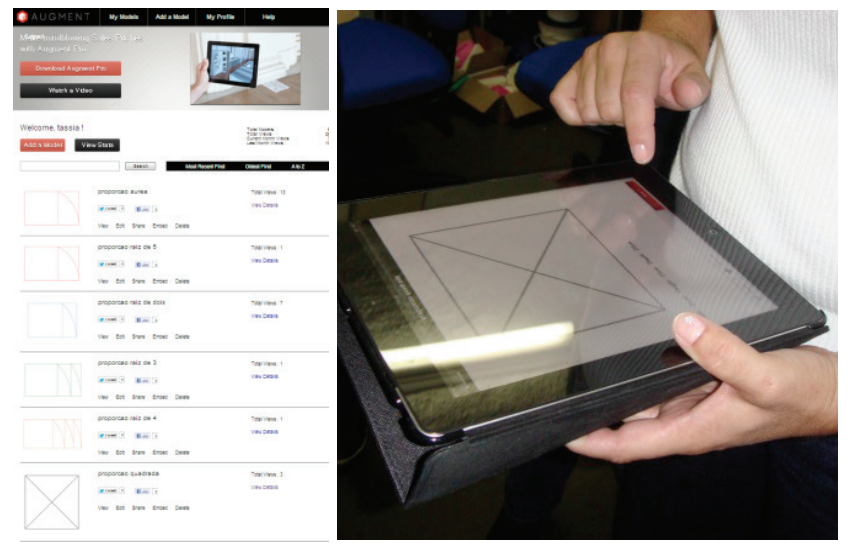

Figura 4: À esquerda, interface do repositório do aplicativo Augment com a lista de elementos de proporção estabelecidos, à direita a imagem dos alunos selecionando uma das proporções. Fonte: Site do aplicativo: (http://augmentedev.com/); foto, autoras.

Para acionar o sistema faz-se necessário imprimir o QRcode. Este é criado pelo próprio aplicativo, para cada uma das figuras depositadas em seu repositório. Com o código impresso basta dirigir a câmera dos dispositivos móveis para que ela o identifique e disponibilize o modelo. As figuras em RA podem ser escalonadas 
através de movimentos táteis, permitindo assim o controle necessário para realizar as análises.

\section{Etapa de Experimentação em contexto educativo:}

Os estudos para inserir as tecnologias de visualização avançadas, como a RA, para as análises de proporção em contexto educativo, começaram no segundo semestre de 2012. Tais estudos foram desenvolvidos junto à atividade de estágio docente no âmbito da disciplina referida anteriormente. Estágio este realizado pela primeira autora deste trabalho, como parte das atividades de mestrado, junto ao PROGRAU/FAURB/UFPel.

O processo de experimentação do proporcionômetro digital incluiu toda sequencia, de aprendizado, estabelecida anteriormente no âmbito da disciplina em questão. Desta maneira o processo foi constituído em três etapas:

\section{a) Construção do proporcionômetro tradicional:}

A partir da representação gráfica de retângulos, cujas relações entre os lados ficam estabelecidas pela proporção áurea, raiz de 2 , de 3 e de 5 os estudantes dimensionam as hastes do proporcionômetro. Cada uma delas associadas a uma cor para facilitar as aferições. Utiliza-se de material transparente como o acetato, para facilitar a sobreposição às imagens. Deve-se destacar que um dos limites deste instrumento se percebe quando as dimensões a serem analisadas ultrapassam à capacidade de abertura das hastes.

\section{b) Proporcionômetro no espaço virtual:}

O proporcionômetro no espaço virtual está sendo configurado através de qualquer programa de desenho vetorial. Os estudantes representam digitalmente os retângulos com proporções determinadas, sobrepondo-os às imagens digitais ou digitalizadas. As vantagens do método são: a precisão; a irrestrição quanto às dimensões; e, a agilidade, especialmente quando se automatiza o processo a partir da configuração dos retângulos em elementos parametrizados.

c) Proporcionômetro em realidade aumentada: proporcionômetro digital.

O proporcionômetro digital está permitindo então a conexão do físico com o virtual através da realidade aumentada. Está sendo utilizado para a análise de documentação arquitetônica, como plantas baixas e fachadas. E, diferenciando-se dos métodos anteriores, está permitindo as análises diretamente sobre a visualização da obra, no espaço real. Essas facilidades tem trazido uma maior agilidade no processo de análise. Porém em termos de precisão, para que se compare ao que se pode obter com o método anterior, diretamente no espaço virtual, faz-se necessária alguma prática de uso. Isto para poder garantir determinada estabilidade para sujeitar o dispositivo móvel durante a análise. Este processo pode ser demostrado à esquerda e acima na figura 5. E, nesta mesma figura podem ser observadas as capturas de telas que resultam da análise de uma fachada, no caso, sobre a fachada do Partenon, 447-432 a.C.

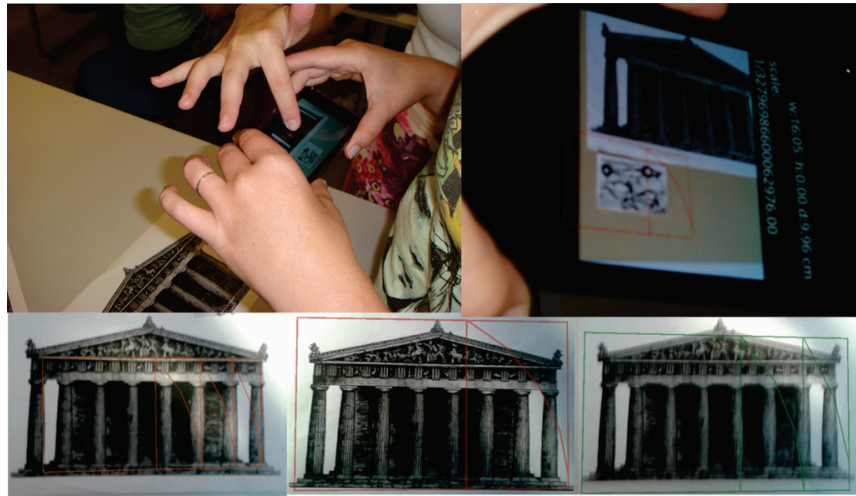

Figura 5: Acima, utilização do proporcionômetro; abaixo, imagens capturadas da tela do dispositivo após sobreposição. Fonte:autoras.

Comparando-se ao uso do proprorcionômetro tradicional, o digital avança de maneira significativa. Através de um movimento tátil pode-se adequar à escala da forma de maneira ilimitada. O processo de captura de tela permite registrar automaticamente cada instante de análise. Movimentos táteis de rotação permitem também agilizar a sobreposição a cada parte do elemento a ser analisado, como tentam demonstrar as imagens da Figura 6.

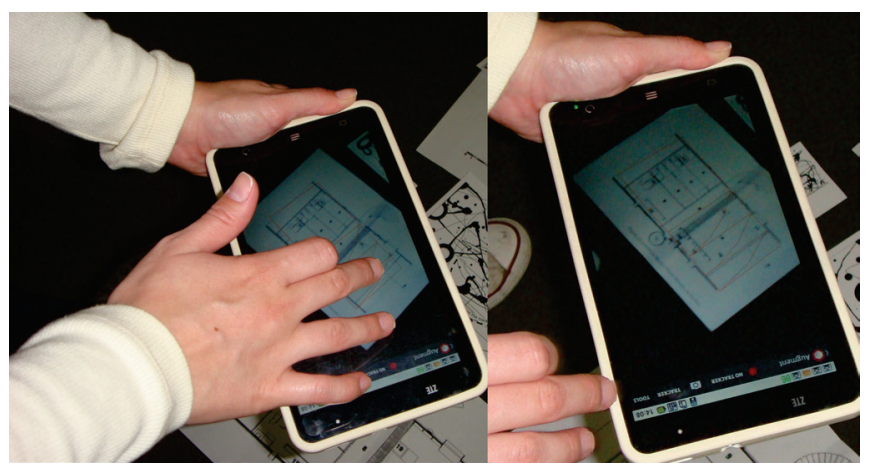

Figura 6: ilustração da utilização do proporcionômetro digital pelos estudantes. Fonte: autoras.

Esta experiência de uso do proporcionômento digital, em realidade aumentada, tem sido validada também em contextos de pósgraduação, junto à disciplina de Modelagem Visual I, do Curso de Especialização em Gráfica Digital/FAURB/UFPel, e junto à atividades de extensão, de acesso à comunidade em geral.

\section{Resultados e Discussões}

Como resultado principal deste estudo se tem um dispositivo que consiste na sobreposição de retângulos com proporções determinadas para análise de arquitetura através de um aplicativo em Realidade Aumentada. Disponível gratuitamente na web para uso em tablets e smartphones. O dispositivo permite visualmente identificar possíveis relações proporcionais em desenhos de vistas ortogonais, ou de maneira aproximada de edifícios físicos.

Como resultados secundários se tem a sistematização da utilização deste aplicativo em contextos de graduação, em estágios iniciais de formação em arquitetura, de pósgraduação e de extensão. Nestes dois últimos contextos, incluindo a formação de 
estudantes, professores e profissionais, especialmente de arquitetura, artes e design.

\section{Considerações Finais}

O estudo aponta métodos e ferramentas para análise de obras de arquitetura sob o conceito de proporção.

Registrou-se neste trabalho o desenvolvimento de um dispositivo que através de realidade aumentada permite realizar análises gráficas de obras de arquitetura. Pode também ser utilizado em outras áreas, tais como design, artes plásticas e até mesmo em dentística.

A ferramenta se utiliza apenas de softwares gratuitos, facilitando seu uso em ações de ensino e aprendizagem.

O instrumento contribui, indiretamente, à percepção de que no processo de configuração formal existem várias ações objetivas e planejadas. Estimula a reflexão sobre a criação da forma, a partir da compreensão formal de estratégias projetuais já estabelecidas.

Além disto, considera-se que a incorporação de técnicas de realidade aumentada em estágios iniciais de formação promove a exploração e apropriação de recursos de representação e visualização avançados. Com isto, poderá abrir novas perspectivas de uso destas tecnologias como apoio ao processo projetual.

\section{Agradecimentos}

Agradecemos à CAPES pelo apoio dado a esta pesquisa através de bolsa de mestrado. Ao Projeto ALFA-GAVIOTA, Grupos Acadêmicos para a Visualização Orientada à Tecnologias Apropriadas, pelo financiamento do laboratório de tecnologia avançada situado no GEGRADI/FAURB/UFPEL (Grupo de Estudos de Ensino e Aprendizagem de Gráfica Digital), e ao PROGRAU/UFPel (Programa de Pós-Graduação em Arquitetura e Urbanismo).

\section{Referências}

Borda, A. S., Pires, J. F. \& Vasconselos, T. B.(2012) O desenho (didático) para o insight in: xvi congresso da sociedade iberoamericana de gráfica digital, 2012, fortaleza. Sigradi Fortaleza. Br., Fortaleza. Fortaleza: Expressão Gráfica e Editora. p.277 - 280

Clark, R. H, \& Pause, M. (1997). Arquitectura: temas de composición, México, Gustavo Gili.

Ching, F. D. K. (1998). Forma espaço e Ordem. São Paulo: Martins Fontes,

Doczi, G. (1990). O poder dos limites: harmonias proporções na natureza. São Paulo: Mercuryo.

Elam, K. (2001). Geometry of design: studies in proportion and composition. New York: Princeton Architectural Press.

Heylighen, A. \& Neuckermans, H. F. (2000). Dynamo: A Dynamic Architectural Memory On-line, Journal of Educational Technology \& Society, vol 3, pp. 86-95. Disponível em: http://www.ifets.info/journals/3_2/heylighen.html

Kirner, C., Kirner, T.G. (2008) Virtual Reality and Augmented Reality Applied to Simulation Visualization. El Sheikh, A.A.R.; Al Ajeeli, A.; Abu-Taieh, E.M.O.. (Ed.). Simulation and Modeling: Current Technologies and Applications. 1 ed. Hershey-NY: IGI Publishing, Retrieved from : http://www.igiglobal.com/Bookstore/Chapter.aspx?Titleld=28994

Krier, R. (1993). Architectural Composition. New York: Rizzoli.

Levin, I. E. (2011). The updated application of the golden proportion to dental aesthetics. Aesthetic dentistry today. Vol. 5 N.3.

Pires, J. F., Nunes, C., Vasconcelos, T. B., Borda, A.B.A.S. (2011) Trajetórias de geometria na arquitetura In: XX Simpósio Nacional de Geometria Descritiva e Desenho Técnico e IX International Conference on Graphics Engineering for Arts and Design, 2011, Rio de Janeiro: UFRJ, Escola de Belas Artes. v.1. p.1-12

Rocha, A. M. D. J.(2011). Divina Proporção: Aspectos Filosóficos, Geométricos e Sagrados da Seção Áurea. Fortaleza: Expressão Gráfica.

Sanz, M. A. \& Moratalla, A. (1999). Simetría. Serie Geometría y Arquitectura II, Cuadernos de Apoyo a la Docencia del Instituto Juan de Herrera. Madri: Publicaciones de la Escuela Superior de Arquitectura de Madrid.

Silva, E.(1984). Uma introdução ao projeto arquitetônico. Porto Alegre: Ed. da Universidade, UFRGS. 